\title{
FULLY NON-LOCAL SUPER-RESOLUTION VIA SPECTRAL HASHING
}

\author{
Emmanuel d'Angelo, Pierre Vandergheynst \\ Ecole Polytechnique Fédérale de Lausanne (EPFL) \\ Signal Processing Laboratory
}

\begin{abstract}
Super-resolution is the task of creating an high resolution image from a low resolution input sequence. To overcome the difficulties of fine image registration, several methods have been proposed exploiting the non-local intuition, i.e. any datapoint can contribute to the final result if it is relevant. These algorithms however limit in practice the search region for relevant points in order to lower the corresponding computational cost. Furthermore, they define the non-local relations in the high resolution space, where the true images are unknown.

In this work, we introduce the use of spectral hashing to efficiently compute fully non-local neighbors. We also restate the superresolution functional using fixed weights in the low resolution space, allowing us to use resolution schemes that avoid many artifacts.
\end{abstract}

Index Terms - Super-resolution, non-local regularization, spectral hashing, image sequence

\section{INTRODUCTION}

Super-Resolution (SR) is the task of creating an high resolution image from a sequence of several lower resolution frames. It has many purposes, such as producing high quality stills from a video sequence or upscaling a movie to an higher resolution. The main intuition to SR methods is to exploit subpixel motions between the frames of the input sequence to infer the missing data on the target high resolution grid.

While initial work involved the fusion of several image spectra [], this approach was then replaced by an inverse problem formulation, which is less sensitive to noise and does naturally handle arbitrary motion. In this context, SR can be stated as an inverse problem, where the Low Resolution (LR) image sequence $\left(\mathbf{I}_{L R}^{k}\right)_{k=0}^{n-1}$ is obtained by applying a blur $B$, a downsampling $D$ and geometric warping $W_{k}$ to the unknown High Resolution (HR) image $\mathbf{I}_{H R}$ :

$$
\mathbf{I}_{H R}=\underset{\mathbf{I}_{H R}}{\operatorname{argmin}} \sum_{k=0}^{n-1} \rho\left(\mathbf{I}_{L R}^{k}, W_{k} D B \mathbf{I}_{H R}\right)+2 \lambda \kappa\left(\mathbf{I}_{H R}\right),
$$

The function $\rho(\cdot, \cdot)$ measures the error between the $k$-th frame in the sequence and the solution, while $\kappa(\cdot)$ penalizes solutions that are too far away from the chosen prior.

Standard choices are the $L_{2}$ norm for the error term $\rho(\cdot)$, and the Tikhonov regularization : $\kappa\left(\mathbf{I}_{H R}\right)=\left\|\nabla \mathbf{I}_{H R}\right\|^{2}$, for the prior. Eq. (1) can then be solved using various frameworks : gradient descent, Iterated Back-Projection (IBP) [1], maximum likelihood estimation [2], etc. (see for example [3] for a review).

Although they lead to well known resolution schemes, these choices have inherent drawbacks. The $L_{2}$ norm is not robust to misalignements between the images. Also, the Tikhonov regularization is known to produce over-smoothed results by penalizing abrupt changes in the gradient of the solution, actually blurring the boundaries of the objects.

Hence, further works [4, 5] considered using the more robust $L_{1}$ norm and the Total Variation (TV) regularization to produce sharper outputs. However, these methods still rely on the accuracy of the motion estimation step.

\section{NON-LOCAL SUPER-RESOLUTION}

\subsection{Related work}

To bypass the limitations of motion estimation methods, recent works take advantage of example-based regularization, successfully introduced by [6] for the SR of a single image. This algorithm however required the training of a Markov random field on a huge database, limiting its practical interest.

Since a movie contains many redundancies, a subsequent work [7] followed the same intuition as the Non-Local Means (NL-Means) image and movie denoising algorithm [8]. The intuition at the heart of NL-Means is to exploit self-similarities anywhere in the image. Pixels are described by a patch, which is simply all the values comprised in a small square neighborhood. If two patches are very similar, then the two pixels are very likely to represent the same phenomenon and should be exploited together, even when they are spatially far from each other.

Hence, the algorithm in [7] uses non-local averaging when fusing together the LR frames interpolated to the final resolution. However, the authors limit the search of self similarities to a small learning window around each pixel to limit the computational overhead. In [9], the authors adapt this approach to the IBP algorithm, again considering non-locality as a post-processing constraint on the upscaled images, and within the limits of a search window.

In this work, we propose to enforce the non-local constraints on the low resolution images (Sec. 2.2). This has two main advantages. First, the weights are fixed by the input LR sequence, which allows to compute the gradient of the non-local error term. Second, since there are less LR pixels, this makes less weights to compute. Furthermore, we propose to use an efficient image hashing algorithm called Spectral Hashing [10] to sort all the input LR patches in a single table, hence leveraging fully non-local SR at a moderate computational cost.

\subsection{Variational TV non-local super-resolution}

We consider that the immutable, and hence reliable, non-local information is contained in the LR frames. Consequently, we do not proceed with a standard SR process followed by an NL-Means like enhancement step, but compute instead a non-local error on the LR 
frames. Hence, our SR functional to be minimized is :

$$
\mathbf{I}_{H R}=\underset{\mathbf{I}_{H R}}{\operatorname{argmin}} \sum_{i=0}^{k}\left\|\mathbf{I}_{L R}^{k}-W_{k}^{N L} D B \mathbf{I}_{H R}\right\|_{2}^{2}+2 \lambda T V\left(\mathbf{I}_{H R}\right) .
$$

Note that $W_{k}^{N L}$ is not a geometric warping anymore, but a weighting matrix. Writing $\mathbf{x}_{i}^{k}$ the patch extracted at the location $i$ in the image $k, W_{k}^{N L}$ is defined by :

$$
W_{k}^{N L}(i, j)=\frac{1}{K_{i}^{k}} \exp \left(-\frac{\left\|\mathbf{x}_{i}^{*}-\mathbf{x}_{j}^{k}\right\|^{2}}{h^{2}}\right),
$$

where the superscript $*$ is the index of the reference LR frame to be upscaled. The constant $K_{i}^{k}$ ensures that each line of $W_{k}^{N L}$ sums to 1. The parameter $h$ is a similarity parameter : for small values of $h$, a candidate patch $\mathbf{x}_{j}$ needs to be very similar to the reference patch $\mathbf{x}_{i}^{*}$ to have a significant contribution.

Following [4, 7], we solve Eq. (2) in two steps iteratively:

1. we first look for an HR blurred estimate $\mathbf{Z}_{H R}=B \mathbf{I}_{H R}$ using non-local back-projection ;

2. then, we compute $\mathbf{I}_{H R}$ by TV deblurring of $\mathbf{Z}_{H R}$.

The TV deblurring subproblem is solved using the Monotonous FISTA (MFISTA) algorithm described in [11].

Our specific form of the SR objective function in Eq. (2) calls for several comments :

- since the weight matrices $W_{k}^{N L}$ are fixed throughout the minimization process, it is possible to define the gradient of the non-local error. Hence, we can use a procedure similar to the FISTA algorithm [11] to iteratively solve the two steps nonlocal error minimization - TV minimization. This minimization process has the advantages of faster convergence and a tighter control of the solution ;

- the normalization of each line of the matrix $W_{k}^{N L}$ leads to a straightforward interpretation of non-local SR as a standard SR process with a probabilistic motion estimation instead of the usual univocal motion model : each line $W_{k}^{N L}(i, \cdot)$ is indeed the motion probability density of the pixel $i$ with respect to the $k$-th image ;

- finally, remark that it is possible to separate the constant from the similarity score in Eq. (3). The matrix $W_{k}^{N L}$ can then be written as the product of a diagonal matrix (storing the constants $\frac{1}{K_{i}^{k}}$ ) by a similarity matrix. This will be useful for the Spectral Hashing (Sec. 2.3).

\subsection{Patch Spectral Hashing}

The bottleneck of non-local algorithms is the huge number of computations needed to compute the matrices $W_{k}^{N L}$. In the context of denoising, it is possible to pre-compute some values (integral images, pre-selection criterion) to reduce the final cost. These techniques however can hardly be used in our case, since we need to find the nearest neighbors to a given patch, and not infer a final value.

This is a problem of nearest neighbors in high dimensions: typical patch sizes are 25 or 49 (5-by-5 or 7-by-7 patches). A naive approach like Principal Component Analysis (PCA) followed by sorting in a $k d$-tree does not help, because the performance of $k d$-trees quickly decreases when the dimension of the space is greater than 5, which is too small to correctly describe a patch.
We considered instead hashing based techniques. Hash tables are highly efficient : the access to the bucket containing a given element is a simple array lookup, hence done in constant time. The main problem then becomes to design a sorting efficient and computationally fast code to distribute the patches over the table.

Recently, Locality Sensitive hashing (LSH) [12] has become very popular for high dimensional data. However, it relies on the choice of several random projections. These projections need to be carefully chosen to ensure that they cover the whole space spanned by the data. This makes LSH unsuitable to sort image patches, since patches in a limited number of locations : many of the random projections become useless.

On the other hand, Spectral Hashing (SH), introduced in [10], was specifically designed to produce compact codes grouping images or parts of images by similarity. Let us consider first scalar points uniformly distributed in the interval $[a, b]$, and a similarity matrix $\mathcal{C}$ defined by :

$$
\mathcal{C}(i, j)=\exp \left(-\frac{\left(x_{i}-x_{j}\right)^{2}}{h^{2}}\right)
$$

which is our patch similarity measure (excluding the normalization constant, as explained in the previous section). Under these hypotheses, one has a closed form solution for the eigenfunctions and eigenvalues of $\mathcal{C}[13]$ :

$$
\begin{aligned}
\Phi_{k}(\mathbf{x}) & =\sin \left(\frac{\pi}{2}+\frac{k \pi}{b-a} x\right), \\
\lambda_{k} & =1-\exp \left(\frac{h^{2}}{2}\left|\frac{k \pi}{b-a}\right|^{2}\right) .
\end{aligned}
$$

Then, retaining the $k$ the smallest eigenvalues, SH simply consists in thresholding the $k$ corresponding eigenfunctions to form binary codes by concatenation. In the case of vector data (such as image patches) of dimension $d$, one can see that $\exp \left(\frac{\left(\mathbf{x}_{i}-\mathbf{x}_{j}\right)^{2}}{h^{2}}\right)=$ $\prod_{l=0}^{d-1} \exp \left(-\frac{\left(\mathbf{x}_{i}^{*}(l)-\mathbf{x}_{j}^{k}(l)\right)^{2}}{h^{2}}\right)$. Hence, one can simply apply the scalar result on each component and the take the product.

While the assumption of uniform distribution seems to be restrictive, SH is surprisingly robust to its violations [10]. Furthermore, the PCA basis provides a coordinate system in which the data follows roughly this distribution along each axis. Finally, this gives us a simple algorithm to compute binary codes associated to a set of patches :

1. find the principal components of the patches from the LR frames. They form the reference (or learning) data;

2. compute the single-dimension eigenfunctions (Eq. (5)) ;

3. retain the $k$ eigenfunctions corresponding to the smallest scalar eigenvalues given by Eq. (6).

The concatenation of the binary thresholded eigenfunctions gives the code associated to any patch $\mathbf{x}$, which in turn corresponds to a bucket index in the hash table. At runtime, one simply needs to project the current patch onto the PCA basis (a matrix-vector multiplication), then threshold $k$ obtained by Eq. (5) values which are also fast to compute. This returns all the patches that have a Hamming distance of 0 with the query patch, which is usually enough. If one wants to find more neighbors, then simple bit offsets produce the codes of the buckets with Hamming distance 1 .

Fig. 1 shows the outputs of several queries (with Hamming distance 0 ) using codes of 8 and 16 bits. Note how the nearest neighbors 
concentrate along geometrically and radiometrically similar image features. Codes of length 16 bits seem to be a good compromise between finding very similar patches while not being too specific. The computations are very fast: each patch is read only twice during the training, then the lookup procedure simply projects the query patch on the PCA basis and reads an index in a table.
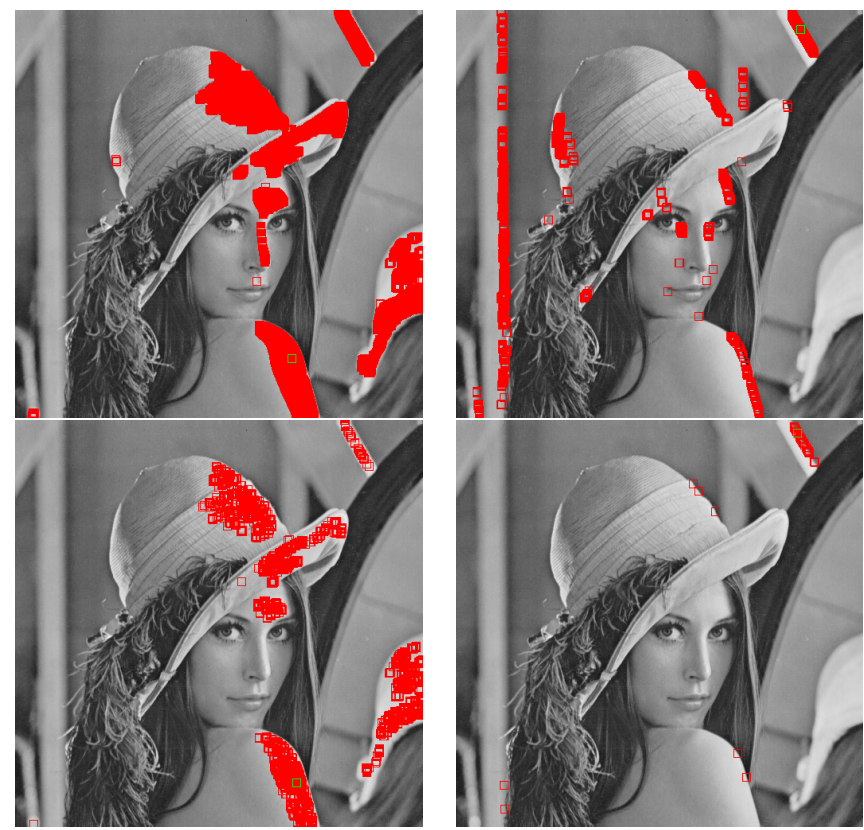

Fig. 1. Query examples by Patch Spectral Hashing. The green square is the query. Red squares are the patches of the same bucket (Hamming distance 0 ) in the hash table, whose similarity score is greater than 0.5 . The length of the codes is 8 bits in the top row, 16 bits in the bottom row.

\subsection{Non-local super-resolution algorithm}

Putting Sec. 2.2 and 2.3 together, we obtain the SR algorithm :

Initialization : 1. Form all the patches from the LR sequence, and compute their PCA.

2. Compute the desired number of eigenfunctions of the similarity matrix.

3. Sort all the input patches in a table, using the retained eigenfunctions to obtain binary codes.

Super-Resolution : 1 . Select the frame to upsample and form an initial estimate by interpolation.

2. Apply the degradation model (blur and downsampling) to the current solution.

3. For each point in the downsampled solution, compute the non-local error using the corresponding patch in the selected frame and its non-local neighbors retrieved by $\mathrm{SH}$.

4. Back-project the non-local error and deblur the updated solution with MFISTA.

Note that, since the hash table contains patches from the whole input sequence, our algorithm is fully non-local : we do not limit the search of relevant patches to a space-time search window. Furthermore, unlike bilateral regularization, there is no additional attenuation factor due to the space or time distance between the patch to update and its neighbors.

\section{EXPERIMENTS}

\subsection{Implementation details}

The algorithm described in Sec. 2.4 is implemented on a standard laptop using $\mathrm{C}++$. Although a Matlab implementation of Spectral Hashing is available online ${ }^{1}$, we did re-implement it using the OpenCV library.

In the nearest neighbor search, we did not use all the patches returned by a query, but only those with a similarity score above 0.9 . The parameter $h$ is fixed depending on the noise level of the input sequence. For clean movies without noise, we used a small value of 0.08 , and values between 0.5 and 1 for noisy inputs. In all our experiments, we have chosen patches of size 5-by-5 pixels.

The initialization of the algorithm is very fast : computing the PCA of a whole LR sequence is the longest part and takes only a few seconds on a laptop for the patch size considered. Then, we used a naive implementation of the algorithm described in Sec. 2.4. Note however that the operations for each pixel are independent : this parallelism can be exploited to design faster multithreaded implementations.

\subsection{Super-resolution results}

We have tested the proposed algorithm on several sequences and reference data available on the internet ${ }^{23}$. Fig. 2 shows the interest of our functional : having fixed weights on the LR input avoids the apparition of the high frequency bright artifacts around the mouth. On the other hand, since we have numerous non-local neighbors from the whole sequence, this is counter-balanced by a more pronounced visual blur with respect to [7].

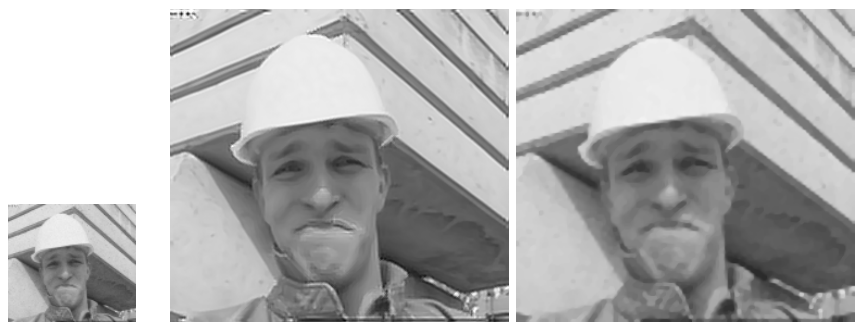

Fig. 2. Comparison with related work. Left : original image. Middle: result of Generalized Non-Local Means [7] for a zoom factor of 3 along each dimension. Right : our result. Our minimization scheme avoided the artifacts around the mouth. While we used a fully non-local approach, there are very few artifacts coming for learning the defects of other frames in the sequence.

Fig. 3 illustrates the application of our algorithm to other noise free sequences.

Finally, since accurate and near real-time optical flow algorithms are becoming available [5], one may wonder why we should still perseverate with non-local SR. If there is only one reason, it should be to

\footnotetext{
${ }^{1}$ http://www.cs.huji.ac.il/ yweiss/SpectralHashing/

${ }^{2}$ http://www.cs.technion.ac.il/ matanpr/Research.html

${ }^{3}$ http://users.soe.ucsc.edu/ milanfar/software/sr-datasets.htm
} 


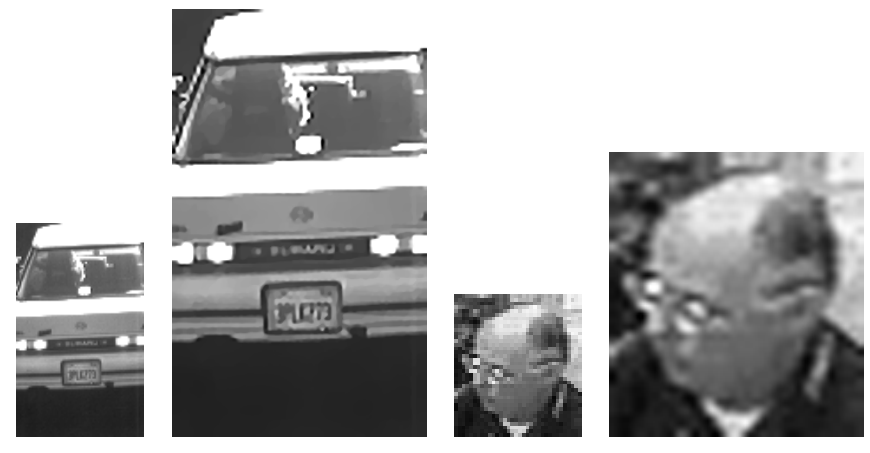

Fig. 3. Results in the noise free case. In both experiments, we used a zoom factor of 3 along each dimension and patches of size 5-by-5 pixels.

deal with noisy sequences. In the presence of noise, the precision of optical flow diminishes quickly. Non-local algorithms, on the other hand, are able to integrate information all along the sequence without explicitly needing any motion estimation, and are designed to naturally deal with independent random noise. This is demonstrated in Fig. 4, using either wider patches or relaxed similarity conditions.

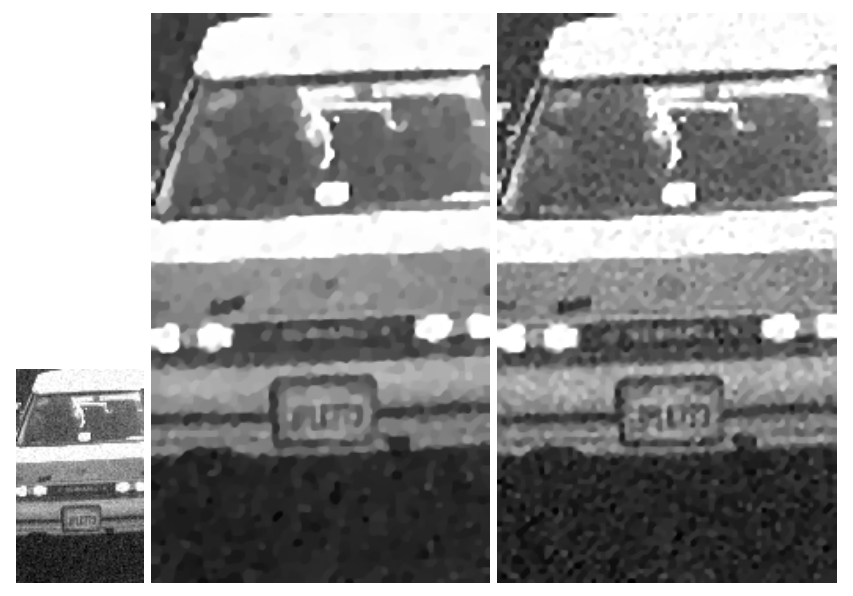

Fig. 4. Noisy example. The input sequence was corrupted with a Gaussian noise of variance 0.05 (the images were rescaled between 0 and 1). In the first case (middle image), we took wider patches (of size 7-by-7 pixels) to make the patch comparison process more robust, which also removed some details with the noise. In the second experiment (right image), we considered a lower threshold to declare a reference patch meaningful, preserving more detail.

\section{CONCLUSION AND FUTURE WORK}

In this work, we have demonstrated the interest of spectral hashing for non-local methods, and applied it to the super-resolution problem. Spectral hashing allows the development of fully non-local algorithms, hence exploiting all the information of the input sequence, while maintaing a low computational complexity.

We also presented a new non-local SR functional, where the non-local weights are fixed. Hence, we can then compute the gradient of the functional and insert it inside algorithms where the op- timization path is controlled, preventing from the apparition of spurious artifacts. Future work will include two main directions : first, the adaptation of the proposed algorithm to the single image SR task, when no input sequence is available. Second, we also plan to apply spectral hashing to previously proposed non-local algorithms, starting with inpainting [14], to study the effects of full non-locality on these specific problems: as can be seen from our results, there is clearly a trade-off to be found between the amount of non-locality and the over-regularization of the results.

\section{REFERENCES}

[1] M. Irani and S. Peleg, "Improving resolution by image registration," CVGIP: Graphical models and image processing, vol. 53, no. 3, pp. 231-239, 1991.

[2] D. Capel and A. Zisserman, "Computer vision applied to super resolution,” IEEE Signal Processing Magazine, vol. 20, no. 3, pp. 75- 86, 2003.

[3] S.C. Park, M.K. Park, and M.G. Kang, "Super-resolution image reconstruction: a technical overview," IEEE Signal Processing Magazine, vol. 20, no. 3, pp. 21- 36, 2003.

[4] S. Farsiu, M.D. Robinson, M. Elad, and P. Milanfar, "Fast and robust multiframe super resolution," IEEE Transactions on Image Processing, vol. 13, no. 10, pp. 1327 - 1344, 2004.

[5] D. Mitzel, T. Pock, T. Schoenemann, and D. Cremers, "Video super resolution using duality based TV-L1 optical flow," Pattern Recognition, pp. 432-441, 2010.

[6] W.T. Freeman, T.R. Jones, and E.C. Pasztor, "Example-based super-resolution," Computer Graphics and Applications, vol. 22, no. 2, pp. 56-65, 2002.

[7] M. Protter, M. Elad, H. Takeda, and P. Milanfar, "Generalizing the nonlocal-means to super-resolution reconstruction," IEEE Transactions on Image Processing, vol. 18, no. 1, pp. 36 - 51, 2009.

[8] A. Buades, B. Coll, and J.-M. Morel, "Nonlocal image and movie denoising," International journal of computer vision, Jan 2008.

[9] W. Dong, L. Zhang, G. Shi, and X. Wu;, "Nonlocal backprojection for adaptive image enlargement," 16th IEEE International Conference on Image Processing (ICIP), pp. 349 352, 2009.

[10] Y. Weiss, A. Torralba, and R. Fergus, "Spectral hashing," Advances in neural information processing systems, vol. 21, pp. 1753-1760, 2009.

[11] A. Beck and M. Teboulle, "Fast gradient-based algorithms for constrained total variation image denoising and deblurring problems," IEEE Transactions on Image Processing, vol. 18, no. 11, pp. 2419 - 2434, 2009.

[12] A. Andoni and P. Indyk, "Near-optimal hashing algorithms for approximate nearest neighbor in high dimensions," 47th Annual IEEE Symposium Foundations of Computer Science, 2006. FOCS '06., pp. 459-468, 2006.

[13] B. Nadler, S. Lafon, R.R. Coifman, and I.G. Kevrekidis, "Diffusion maps, spectral clustering and reaction coordinates of dynamical systems," arXiv, 2005.

[14] E. D'Angelo and P. Vandergheynst, "Towards unifying diffusion and exemplar-based inpainting," in 17th IEEE International Conference on Image Processing (ICIP), 2010. 